\title{
Comparison of different Mo/Au TES designs for radiation detectors
}

\author{
Carlos Pobes $^{1} \bullet$ Lourdes Fàbrega $^{2}$ • Agustín Camón ${ }^{1} \bullet$ Pavel \\ Strichovanec $^{2}$ • Javier Moral-Vico ${ }^{2}$ - Nieves Casañ-Pastor ${ }^{2} \bullet$ \\ Rosa M. Jáudenes ${ }^{2} \bullet$ Javier Sesé $^{3}$
}

1. Instituto de Ciencia de Materiales de Aragón, CSIC-Universidad de Zaragoza, 50009 Zaragoza, Spain

2. Institut de Ciència de Materials de Barcelona, CSIC, 08193 Bellaterra, Spain

3. Instituto de Nanociencia de Aragón, Universidad de Zaragoza, 50018 Zaragoza, Spain

\begin{abstract}
We report on the fabrication and characterization of $\mathrm{Mo} / \mathrm{Au}$-based Transition Edge Sensors (TES), intended to be used in X-ray detectors. We have performed complete dark characterization using I-V curves, complex impedance and noise measurements at different bath temperatures and biases. Devices with two designs, different sizes and different membranes have been characterized, some of them with a central Bismuth absorber. This has allowed extraction of the relevant parameters of the TES, analyses of their standard behavior and evaluation of their prospects.
\end{abstract}

Keywords Cryogenic Detectors - Transition Edge Sensors •

Microcalorimeters

\section{Introduction}

Superconducting microcalorimeters based on Transition Edge Sensors (TESs) [1,2], are at present the most mature and best performing technology for high resolution single photon spectroscopy, especially for X-rays. TES are considered basic elements in a variety of highly demanding instruments, both on Earth and in space, in fields such as astrophysics, particle physics and materials science. For instance, the detector of the X-IFU (X-ray Integral Field Unit) instrument, the high resolution spectrometer onboard ESA's next X-ray telescope Athena, will be constituted by an array of nearly $4000 \mathrm{Mo} / \mathrm{Au}$ TESs [3]. 


\section{C.Pobes • L.Fàbrega • A.Camón • P.Strichovanec • J.Moral-Vico • N.Casañ-Pastor • R.M.Jáudenes • J.Sesé}

$\mathrm{We}$ are developing $\mathrm{Mo} / \mathrm{Au}$ TESs for X-ray detection. The dark characterization of several devices, with different sizes and shapes, with and without banks, with and without central absorber, and with different membrane size and thickness, has allowed extraction of the basic behavior of our TESs as a function of these parameters, bath temperature and bias point. Results are quite promising and provide guidelines for optimization and tuning of the devices.

\section{Device fabrication}

$\mathrm{Mo} / \mathrm{Au}$ TESs with critical temperature $\mathrm{T}_{\mathrm{c}} \sim 100 \mathrm{mK}$ were fabricated on low stress $\mathrm{Si}_{3} \mathrm{~N}_{4}$ membranes with thickness 0.5 and $1 \mu \mathrm{m}$ and sizes between 250 and $1000 \mu \mathrm{m}$. Mo/Au thicknesses, TES components and deposition techniques were as reported previously [4,5]. Refinement of the photolithography procedures [6] has allowed complete dry etching processing, high reproducibility and transition widths of $1-2 \mathrm{mK}$, even without banks. Normal resistance values $R_{n}$ ranged between 10 and $29 \mathrm{~m} \Omega$.

Fig. 1 left displays an optical image of one of the devices without absorber. Square TES sizes between $100 \times 100 \mu \mathrm{m}^{2}$ and $200 \times 200 \mu \mathrm{m}^{2}$, and a rectangular shape with dimensions $100 \times 140 \mu \mathrm{m}^{2}$ were tested.

Some devices incorporated a central square absorber, either a block or mushroom-shaped, made of electrodeposited bismuth, with typical thickness 4-6 $\mu \mathrm{m}$ (Fig.1 right). The electrolyte used for Bi electrodeposition was NB semiplate 100, from NB Technologies, and was deposited with a constant current. The absorber was defined by conventional photolithography techniques, using a seed layer made of $5 \mathrm{~nm} \mathrm{Ti}$ and $100 \mathrm{~nm} \mathrm{Au}$, both thermally evaporated.

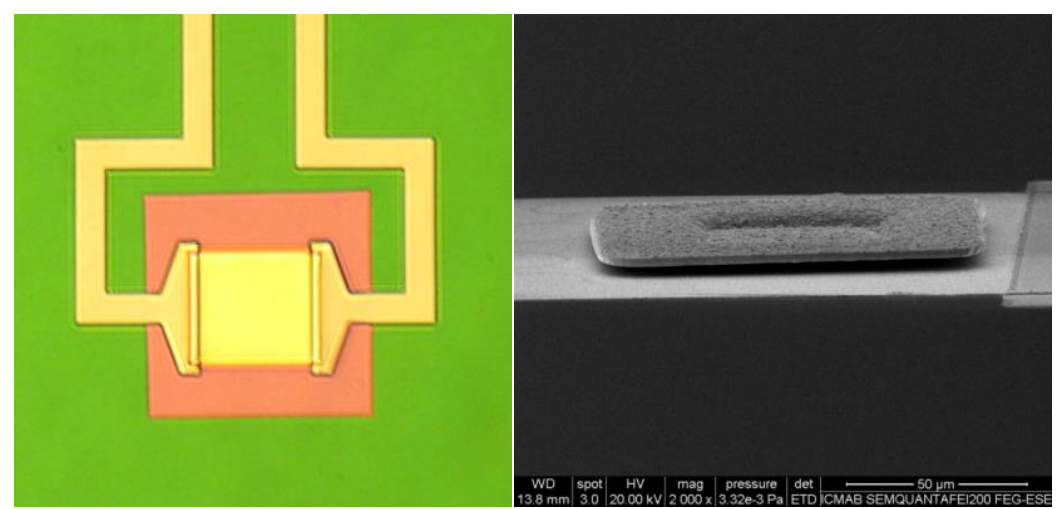

Figs. 1 Optical photograph of a bare TES of dimensions $120 \mu \mathrm{m} \times 120 \mu \mathrm{m}$ on a $250 \mu \mathrm{m} \times 250 \mu \mathrm{m}$ membrane (left) and SEM micrograph of a mushroom shaped $\mathrm{Bi}$ absorber on a TES (right). 


\section{Comparison of different Mo/Au TES designs for radiation detectors}

\section{Characterization}

TESs were characterized through I-V curves, complex impedance and noise measurements under DC bias, using the setup described previously [5]. This complete dark characterization was performed at different bath temperatures $\mathrm{T}_{\text {bath }}$ and bias currents (bias points). Relevant parameters were estimated as is common in literature (see for instance [1] for parameter definition). Thermal parameters (power, thermal conductance and exponent ' $n$ ') were obtained from the I-V curves, recorded with positive and negative current. Extraction of the main TES parameters (heat capacity C, sensitivity to temperature $\alpha$ and current $\beta$, and effective time constant $\tau_{\text {eff }}$ ) was carried out using a single thermal block model (STM), which provides a reasonable approximation for the analyzed devices (most of them had no absorber, and in those with absorber the very low heat capacity of bismuth could be ignored).

Fig. 2 displays the thermal conductance to the bath, for the tested devices, as a function of the radiative area, that is, the TES perimeter times the membrane thickness. A linear dependence is observed, as expected for dominant heat conduction by radiation; obtained exponents ' $n$ ' lie always between 3.2 and 3.4. The behavior and values observed in Fig.2 indicate that $\mathrm{G}$ values as those required for the X-IFU requirements (LPA2 [3]) are reached for TESs close to $100 \times 100 \mu \mathrm{m}^{2}$ on a membrane with thickness of $0.5 \mu \mathrm{m}$ (lower left corner on the graph).

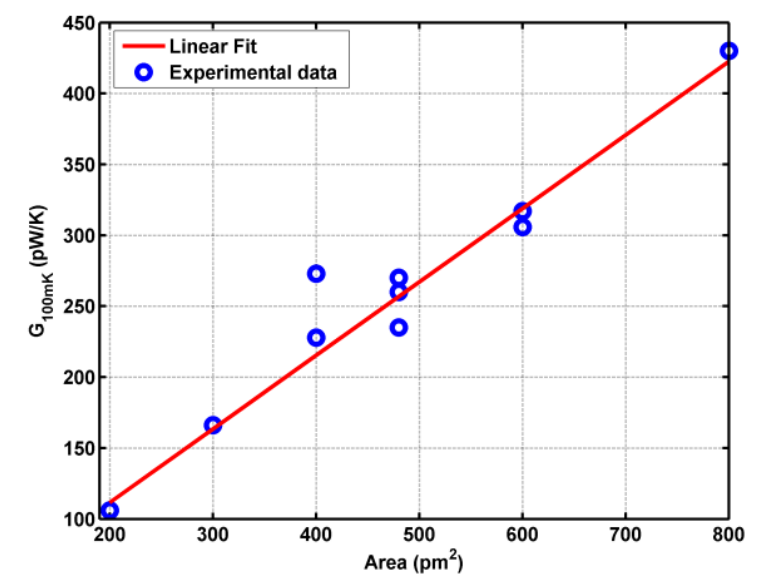

Fig. 2 Thermal conductance scaled at $100 \mathrm{mK}$ as a function of the radiative area, i.e. TES perimeter times membrane thickness. (Color figure online) 


\section{C.Pobes • L.Fàbrega • A.Camón • P.Strichovanec • J.Moral-Vico・ N.Casañ-Pastor • R.M.Jáudenes • J.Sesé}

Fig. 3 displays the sensitivity of the transition resistance to temperature, $\alpha$, as a function of the bias point, at $T_{\text {bath }}=50 \mathrm{mK}$. For most of the tested devices $\alpha$ decreases with increasing bias, displaying values between 50 and 100 at low $\% \mathrm{R}_{\mathrm{n}}$; some TESs show higher values, up to 200 . These values are fully comparable to those reported by other groups $[7,8,9]$. The $\beta$ parameter, characterizing the sensitivity of the transition to current, lies between 0.1 and 1 , also in accordance with other reported values, and below the line delimitating the separation between the weak link and phase slip dominated regimes, described by Bennet et al. [10]. There appears to be a correlation between $\alpha$ and $\beta$ values (higher $\alpha$ values correspond to higher $\beta$ values), as should be expected.
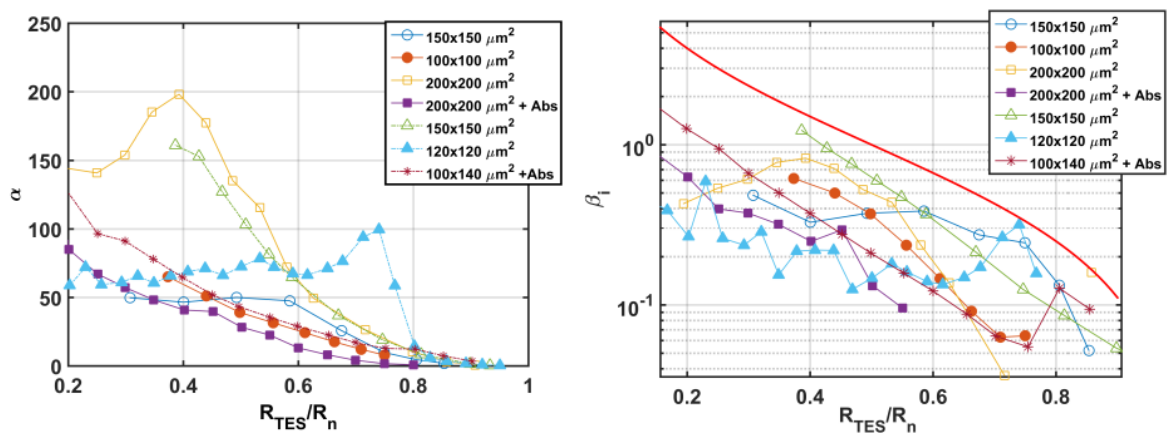

Figs. $3 \alpha$ (left) and $\beta$ (right) at $50 \mathrm{mK}$ along the superconducting transition. Legends indicate TES bilayer dimension. Continuous line in $\beta$ plot indicates weak link and phase slip regime separation [10]. The four upper TESs in the legend have banks, while the other three do not. (Color figure online)
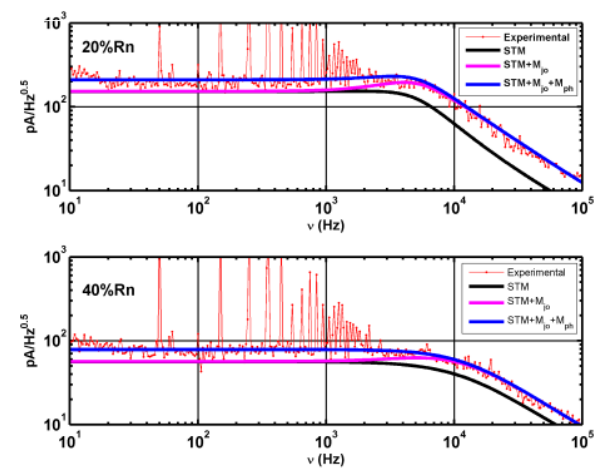

Fig. 4 Two noise spectra of a representative TES $(200 \mu \mathrm{m}$ side with absorber in Figs.3), measured at $\mathrm{T}_{\text {bath }}=50 \mathrm{mK}$ and at biases of $20 \%$ and $40 \% \mathrm{R}_{\mathrm{n}}$, respectively. The solid lines display calculated noise using the experimental TES parameters and the simplest thermal model (STM) [1], as described in the text. (Color figure online) 


\section{Comparison of different Mo/Au TES designs for radiation detectors}

It should be stressed that the application of a magnetic field was not available for measurements reported in this work; thus, it was not possible to cancel any trapped field. It has been lately estimated that remanent fields on the order of 4-5 $\mu \mathrm{T}$ may be present in the setup. This could account for the variability in sensitivity $\alpha$ and $\beta$, but would not change the reported behaviour of the other parameters, which display standard dependences and scale nicely with membrane and TES sizes.

Fig. 4 displays two spectra, representative for our devices, at two different bias points and $\mathrm{T}_{\text {bath }}$ well below $\mathrm{T}_{\mathrm{c}}$. Also shown in the figure are three noise curves, calculated by (i) the simplest model (one thermal block, [1]) using the experimental TES parameters extracted from the complex impedance and I-V data, by (ii) adding the extra Johnson noise term, which has become a standard way of accounting for the so-called unexplained noise [9], and by (iii) including also a phonon-like noise term to account for the extra noise observed still at low frequencies. The two additional noise components just described are parametrized respectively by $\mathrm{M}_{\mathrm{jo}}$ and $\mathrm{M}_{\mathrm{ph}}$ [11]. Fig.4 reveals that the STM reproduces rather well the experimental noise, thereby validating the use of the single thermal block model to extract the relevant TES parameters. Introduction of the extra Johnson noise, as usual, accounts for the bump observed in the spectra at $v \sim 10 \mathrm{kHz}$. Extracted $\mathrm{M}_{\mathrm{j} o}$ values are typically between 1 and 2 , thus indicating that the unexplained noise $[8,9]$ in these TESs is rather low, even though they do not contain normal metal structures. The other noise component, $\mathrm{M}_{\mathrm{ph}}$, which accounts for the low frequency extra noise, displays nearly constant values close to 0.5 for all TES. This component, totally uncorrelated to $\mathrm{M}_{\mathrm{jo}}$, is likely to correspond to an additional thermal fluctuation noise, which could be accounted for by more complex models, with additional thermal blocks [12].

\section{Conclusions}

We have fabricated Mo/Au TES devices with different sizes, geometries and thermal conductances, with and without banks, and with and without central absorbers. Systematic characterization of these devices as a function of bath temperature and bias has allowed extraction of their standard behavior and relevant parameters. We have shown that our TESs display a rather robust and coherent general behavior (notably for thermal parameters), with values close to the state of the art and interesting prospects. Variability in $\alpha$ and $\beta$ may be partly explained by trapped field in our setup and will be further analyzed in future work. It is worth mentioning that Mo/Au TESs with and without banks display identical performances [6], and show rather low excess noise without normal metal structures in both cases. 


\section{C.Pobes • L.Fàbrega • A.Camón • P.Strichovanec • J.Moral-Vico・ N.Casañ-Pastor • R.M.Jáudenes • J.Sesé}

Acknowledgements Work financed by the Spanish Ministerio de Economía y Competitividad (MINECO, projects ESP2014-59306-JIN, ESP201453672-C3-2-P, ESP2016-76683-C3-2-R), the European Space Agency (ESA, CTP Contract "Optimization of a European TES array") and the European Commission (H2020 project AHEAD: Integrated Activities for the high energy astrophysics domain"). Personnel from ICMAB acknowledge financial support from MINECO, through the Severo Ochoa Programme for Centres of Excellence in R\&D (SEV-2015-04969. RMJ wishes to thank MINECO for her FPI contract. We also thank PTB for providing the SQUIDs.

\section{References}

1. K.D.Irwin, C.C.Hilton, "Transition-Edge Sensors in Cryogenic Particle Detection", edited by C.Enss, Springer-Verlag, Berlin Heidelberg, Topics in Applied Physics, vol. 99, pp.63-149, 2005.

2. D.A.Bennett, J.N.Ullom, "Review of superconducting TES for X-ray and g-ray astrophysics", Supercond. Sci. Technol. Vol. 28, 084003, 2015.

3. D.Barret et al., "The Athena X-ray integral field unit (X-IFU)", Proc. SPIE vol 9905, 99052F, 2016.

4. L.Fàbrega et al., "Mo-based proximity bilayers for TES", IEEE Trans. Appl. Supercond. Vol 19, 460, 2009.

5. C.Pobes et al., "Development of cryogenic X-ray detectors based on Mo/Au Transition Edge Sensors"IEEE Trans. Appl. Supercond. Vol 27, 2101505, 2017.

6. P.Strichovanec et al., in preparation.

7. S.J.Smith et al., "Transition-edge sensor pixel parameters design of the microcalorimeters array for the X-ray Integral Field Unit on Athena", Proceed. SPIE, vol. 9905, 99052H, 2016.

8. H.F.C.Hoevers et al., "Comparative study of TiAu-based TES microcalorimeters with different geometries", J. Low Temp. Phys. Vol 151, 94, 2008.

9. J.N.Ullom et al., "Characterization and reduction of unexplained noise in superconducting transition-edge sensors", Appl. Phys. Lett. vol 84, 4206.

10. D.A.Bennett et al., "Resistance in transition-edge sensors: A comparison of the resistively shunted junction and two-fluid models", Phys. Rev. B vol 87, 020508(R), 2013.

11. M.Galeazzi, "Fundamental Noise Processes in TES devices", IEEE Trans. Appl. Supercond. Vol 21, 267, 2011.

12. I.J.Maasilta, "Complex impedance, responsivity and noise of transitionedge sensors: analytical solutions for two- and three-block thermal models", AIP Advances vol. 2, 042110, 2012. 